\title{
Influence of periodontal condition on levels of human beta defensins $I$ and 2 in saliva
}

\begin{abstract}
Many research studies have looked into periodontal disease biomarkers. The aim of this study was to assess salivary levels of human beta defensin 1 and 2 (hBD-1) and (hBD-2) in different periodontal condition. The Periodontal Screening \& Recording (PSR) system, a modification of de Community Periodontal Index Treatment Needs (CPITN), was used to evaluate the periodontal treatment needs of 120 patients without any systemic disease. Salivary levels of hBD-1 and hBD-2 were determined by ELISA. Higher hBD-1 levels were detected in the saliva of patients with a PSR/CPITN index of 3 and $4(7,363 \pm 2,837 \mathrm{pg} /$ $\mathrm{mL})$ and in those with a PSR/CPITN index of 1 and $2(7,750 \pm 3,477 \mathrm{pg} / \mathrm{mL})$ when compared to patients without periodontal disease, with a PSR/CPITN index of $0(5,644 \pm 2,747 \mathrm{pg}$ / $\mathrm{mL} ; P=0.013)$. As with hBD-1, hBD2 levels were also higher in the saliva of patients with a PSR/CPITN index of 3 and $4(310 \pm 98 \mathrm{pg} / \mathrm{mL})$ and a PSR/CPITN index of 1 and 2 $(318 \pm 85 \mathrm{pg} / \mathrm{mL})$ when compared to patients with a PSR $/$ CPITN index of $0(251 \pm 87 \mathrm{pg} / \mathrm{mL}$; $P=0.011)$. In conclusion, compared to patients without periodontal disease, PSR/CPITN index 0 , we verified higher salivary concentrations of hBD-1 and hBD-2 in patients with some degree of periodontal detected by PSR/CPITN between 1 and 4 .
\end{abstract}

Keywords: biomarkers, saliva, periodontal screening and recording, periodontal disease, human beta
Research Article

Volume 6 Issue I - 2018

\author{
Ana Elisa Rodrigues Alves Ribeiro,' Alan \\ Grupioni Lourenço,' Ana Carolina Fragoso \\ Motta, ${ }^{2}$ Marilena Chinali Komesu' \\ 'Department of Morphology, Physiology and Basic Pathology, \\ University of São Paulo, Brazil \\ ${ }^{2}$ Department of Stomatology, Public Oral Health and Forensic \\ Dentistry, University of São Paulo, Brazil
}

\begin{abstract}
Correspondence: Marilena Chinali Komesu, Department of Morphology, Physiology and Basic Pathology, Ribeirão Preto School of Dentistry, University of São Paulo,Via do Café s/n, |4040-900 Ribeirão Preto, SP, Brazil, Tel +55 1633 | 5 40|2, Email mckomesu@usp.br
\end{abstract}

Received: August 22, 2017 | Published: February 26, 2018
Abbreviations: CPITN, communiy periodontal index treatment needs; PSR, periodontal screening \& recording; hBD 1, human beta defensin 1; hBD2, human beta defensin 2; LPS, lipopolysaccharides; TNF- $\alpha$, tumor necrosis factor-alpha; IL-1 $\beta$, interleukin-1 beta; ELISA, enzyme-linked immunosorbent assay; PBS, phosphate buffered saline; ABTS, 2'-azino-bis-3-ethylbenzothiazoline-6-sulfonic acid

\section{Introduction}

Periodontal diseases are the most frequent oral inflammatory reactions, affecting around $90 \%$ of the population worldwide, $, 1,2$ leading to local and systemic morbidity. There is great interest in finding biomarkers of periodontal disease as they could shed further light upon its pathophysiology and also upon its relationship with systemic factors. ${ }^{3}$ Salivary biomarkers may give some important information about unusual cellular activity in periodontal disease, about the effectiveness of periodontal treatment, and also about individual susceptibility to the development of periodontitis. ${ }^{4,5}$ In addition, to monitor disease progression is a highly skilled action, involving various measures like bleeding on probing, probing depth and attachment loss coupled with radiographic observations. Therefore, it would be desirable to develop biomarkers for early detection of periodontal disease and to identify progression because current diagnostic approaches do not reflect current disease activity but simply assess the cumulative effects of historical tissue destruction. ${ }^{6}$ Some of these potential biomarkers include human defensins, which are small cysteine-rich cationic peptides, with antimicrobial and immunomodulatory properties, and are found in different tissues of the oral cavity, including salivary glands and gingival tissues. ${ }^{7,8}$

Recent studies have shown that defensins contribute to antimicrobial defenses, suggesting its capacity to inhibit multiple pathogens in different stages of infection. ${ }^{9}$ Moreover, these peptides seem to play an important role in adaptive immune system, providing immune surveillance by influencing the maturation of dendritic cells, recruiting immature dendritic and $\mathrm{T}$ cells, interacting with chemokine receptor type 6 , and activating these cells by toll-like receptor $4 .^{10}$ Human defensins are classified into alpha, beta, and theta based on their size and cysteine distribution, and six beta defensins have been identified to date, ${ }^{5}$ but only types 1,2 , and 3 are expressed in tissues of the oral cavity. ${ }^{11}$ Human beta defensin 1 (hBD-1) is continuously expressed by epithelial tissues ${ }^{12}$ while human beta defensin 2 (hBD-2) and human beta defensin 3 (hBD-3) are induced by lipopolysaccharides (LPS), peptidoglycans, and inflammatory mediators, such as tumor necrosis factor-alpha (TNF- $\alpha$ ) and interleukin-1 beta (IL-1 $\beta){ }^{13}$

Previous studies suggested hBD-1 and hBD-2 expression in gingival tissue, gingival cervicular fluid, and saliva can be altered in several oral inflammatory conditions, including periodontal disease. ${ }^{14}$ In addition to the continuous search for biomarkers of periodontal disease, simple periodontal screening techniques have been developed for its early detection. The American Dental Association and the American Academy of Periodontology ${ }^{15}$ developed a simple periodontal screening method known as Periodontal Screening and Recording (PSR) index, which has been widely used and accepted for the early detection of periodontal disease. The PSR index is a modification of the Community Periodontal Index of Treatment Needs (CPITN),${ }^{16}$ developed by the World Health Organization to estimate periodontal treatment needs in different population groups. ${ }^{17}$

The present study assumes that salivary levels of hBD-1 and hBD-2 are influenced by patient's periodontal condition and can be helpful for early periodontal disease detection, and, therefore, aims to 
assess the hBD-1 and hBD-2 concentrations in different periodontal conditions detected by the PSR/CPITN index, that is used worldwide for the early detection of periodontal disease.

\section{Materials and methods}

\section{Ethics statement}

The study protocol was approved by the Ethics Committee of the School of Dentistry of Ribeirão Preto, University of São Paulo, Ribeirão Preto, Brazil (CAAE protocol no. 0003.0.138.000-10). All patients signed a written informed consent form for their participation.

\section{Patients}

A total of 120 patients referred to the Periodontology Outpatient Clinic affiliated with the School of Dentistry of Ribeirão Preto, University of São Paulo, were selected. The inclusion criteria were the following: age over 18 years; no history of systemic diseases, such as diabetes, viral hepatitis, HIV infection; systemic bacterial or fungal infections; dentition containing at least 19 teeth; no previous periodontal treatment in the past 6 months; no antibiotic treatment in the past 3 months; and no history of xerostomia and hyposalivation. The patients were evaluated by anamnesis and clinical examination, during which they were asked about their medical and dental history and had a complete physical extraoral and intraoral examination in order to find any oral mucosal abnormalities.

An experienced examiner (AERAR) estimated the PSR/CPITN index, following the guidelines established by the American Dental Association and the American Academy of Periodontology ${ }^{15}$ using a periodontal probe, as recommended by the World Health Organization (WHO-621). A specialized periodontal probe with a ball shaped tip having a $0.5 \mathrm{~mm}$ diameter was used to examine and score six sites per tooth in each patient sextant on a 0 to 4 hierarchical grading scale, identical to CPITN criteria, ${ }^{16}$ with only the highest PSR score per sextant recorded for documentation. ${ }^{17}$ After data collection, patients received oral hygiene instructions, information on their periodontal status and periodontal treatment according to their PSR/CPITN codes. Patients were divided into three different groups based on their periodontal status:

Group 1: Periodontally healthy patients whose six sextants were classified as code 0 ;

Group 2: Patients with at least one sextant scored as code 1 or 2;

Group 3: Patients with at least one sextant scored as code 3 or 4.

\section{Saliva collection}

All patients were instructed not to eat, drink, smoke or brush their teeth at least 1 hour before saliva collection, thereby avoiding contamination risks. Patients were instructed to sit with their heads slightly tilted down and had their saliva sampled from a collector placed below their lower lips. The total amount of saliva collected within a 5 -minute period was approximately $5 \mathrm{~mL}$. The samples were immediately centrifuged $\left(45,000 \mathrm{rpm}\right.$ for $30 \mathrm{~min}$ at $\left.4^{\circ} \mathrm{C}\right)$ and stored at $-80^{\circ} \mathrm{C}$ until analysis.

\section{ELISA}

Salivary levels of hBD-1 and hBD-2 were assessed by enzymelinked immunosorbent assay (ELISA). Human beta defensin 1 peptide (sc65501 - Santa Cruz, Dallas, USA) was used for hBD-1 analysis and the initial serial dilutions were prepared at a concentration of $800 \mathrm{pg} / \mathrm{mL}$. Briefly, $100 \mu \mathrm{L}$ of each concentration was added, in duplicate, per well. The saliva samples were diluted 10 times in phosphate buffered saline (PBS) and transferred in duplicate to ELISA plates $(100 \mu \mathrm{L}$ per well). Different hBD-1 concentrations and saliva samples were placed in polystyrene microplates and kept overnight at $4^{\circ} \mathrm{C}$. After incubation and washing in PBS, nonspecific binding was blocked by adding $200 \mu \mathrm{L}$ of $10 \%$ ovalbumin (albumin from chicken egg whites grade II, Sigma-Aldrich, St Louis, USA) per well for 2 $\mathrm{h}$ at room temperature. After another wash, $100 \mu \mathrm{L}$ of $\mathrm{hBD}-1$ mouse monoclonal antibody (IgG M4-14b-H4 -Santa Cruz, Dallas, USA) diluted 500 times in $10 \%$ ovalbumin was added per well, followed by two-hour incubation at room temperature. After another wash, $100 \mu \mathrm{L}$ of goat anti-mouse IgG-HRP (Santa Cruz, Dallas, USA) diluted 2,000 times in $10 \%$ ovalbumin was added to each well and then incubated for 45 minutes. After another wash, treatment with 2, 2'-azino-bis3-ethylbenzothiazoline-6-sulfonic acid (ABTS) and ABTS enhancer (Sigma-Aldrich, St. Louis, USA) was performed according to the manufacturer's recommendations to detect peroxidase activity. Absorbance was analyzed by an ELISA reader (DIATECH) at 405 $\mathrm{nm}$. ELISA was also used for hBD-2 (hBD-2 ELISA Development Kit - Peprotech, New Jersey, USA) according to the manufacturer's instructions. The saliva samples were diluted 1:2 in PBS and measured at $405 \mathrm{~nm}$.

\section{Statistical analysis}

Prior to this study, the clinical parameters and salivary levels of hBD- 1 and hBD- 2 were determined in five patients from each of the three groups. The results of this pilot study were assessed by Student's $t$ test in order to calculate the sample size, using a 95\% confidence interval and statistical power of $90 \%$. The sample should include 35 patients per group, and we added $10 \%$ to this number, totaling 40 participants in each group. The data obtained from the pilot study were not accounted for in the final statistical analysis.

The data are presented as mean \pm standard deviation. The chisquare test was used to compare gender and number of smokers in the three groups. The Kolmogorov-Smirnov test was used to verify the normality of data distribution. Outliers were not removed from the data analysis. The Kruskal-Wallis test was used for analysis of three different means, followed by Dunn's post-hoc test for assessment of differences between the means obtained for each group. In all cases, a $95 \%$ confidence interval was computed and statistical significance was obtained when $P$ value $<0.05$. All statistical analyses were performed using the GraphPad software (San Diego, CA, USA).

\section{Results}

A total of 120 patients ( 84 women and 36 men) aged 18 to 63 years were assessed as to their periodontal status using the PSR/ CPITN index and as to their salivary levels of hBD-1 and hBD-2. The demographic data and the clinical parameters are summarized in Table 1. The mean age was similar between Groups 1 and 2; however, patients from Group 3 were older than those from Groups 1 and 2 $(P<0.001)$. Also, Group 3 had a lower average number of teeth than Groups 1 and $2(P<0.001)$.

Patients with periodontal disease (Groups 2 and 3) had a higher prevalence of BOP and higher pd than those with no periodontal disease (Group 1) $(P<0.0001)$. No differences in smoking habits were 
found between the groups $(P=0.31)$. Higher salivary levels of hBD-1 and hBD-2 were found in patients with periodontal disease. Patients with a PSR/CPITN index of 1 and $2(7,750 \pm 3,477 \mathrm{pg} / \mathrm{mL})$ and those with a PSR/CPITN index of 3 and $4(7,363 \pm 2,837 \mathrm{pg} / \mathrm{mL})$ had higher concentrations of hBD-1 in their saliva compared to periodontally healthy individuals $(5,644 \pm 2,747 \mathrm{pg} / \mathrm{mL})$. $(P=0.013$; Kruskal-Wallis test). Dunn's post-hoc test showed some differences between Groups 1 and 2 and between Groups 1 and 3, but no difference was found for hBD-1 levels between Groups 2 and 3 (Figure 1).

Table I Demographic data and clinical parameters of three groups of patients according to their periodontal status, determined by the PSR index

\begin{tabular}{|c|c|c|c|c|}
\hline & Group I & Group 2 & Group 3 & $\mathbf{P}$ \\
\hline Number & 40 & 40 & 40 & - \\
\hline Age (years) & $26.7 \pm 8.1$ & $33.4 \pm 13.7$ & $43 \pm 11.2$ & $<.000 I^{*}$ \\
\hline Males (\%) & 25 & 27,5 & 37,5 & 0.4346 \\
\hline Smoking (\%) & 10 & 7,5 & 17,5 & 0.3101 \\
\hline Mean number of teeth & $27.8 \pm 1.8$ & $27.3 \pm 1.1$ & $25.3 \pm 3.6$ & $<0.000 I^{*}$ \\
\hline Sextant healthy - PSR code 0 & 6 & $2.1 \pm 1.4$ & $0.4 \pm 0.7$ & $<0.000 I^{*}$ \\
\hline Sextant BOP - PSR code I & 0 & $3.4 \pm 1.6$ & $0.1 \pm 0.5$ & $<0.000 I^{*}$ \\
\hline Sextant dental calculus - PSR code 2 & 0 & $0.5 \pm 0.7$ & $0.9 \pm 1.4$ & $<0.000$ I* \\
\hline Sextant PD 3.5 to $5.5 \mathrm{~mm}$ - PSR code 3 & 0 & 0 & $2.3 \pm 1.5$ & $<0.0001 *$ \\
\hline Sextant PD >5.5 mm - PSR code 4 & 0 & 0 & $2.1 \pm 1.9$ & $<0.000 I^{*}$ \\
\hline
\end{tabular}
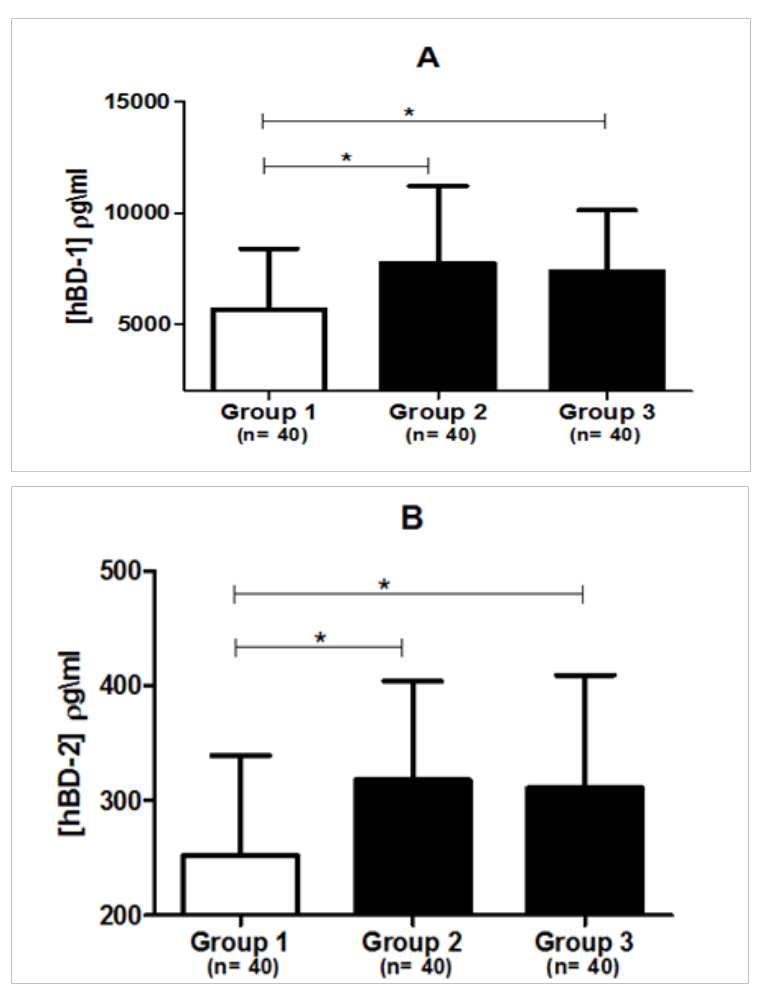

Figure I (A) Salivary hBD-I concentration. (B) Salivary hBD-2 concentration in three different groups based on their periodontal status.

Group I, periodontally healthy patients whose six sextants were classified as PSR code 0; Group 2, patients with at least one sextant scored as PSR code I or 2; Group 3, patients with at least one sextant scored as PSR code 3 or 4.

*Statistical significance in relation to periodontally healthy subjects using Dunn's post-hoc test $(P<0.05)$.

hBD-I, human beta-defensin I; hBD-2, human beta-defensin 2; n, number of experiments.
As with hBD-1, salivary levels of hBD-2 were higher in Group $2(317 \pm 85 \mathrm{pg} / \mathrm{mL})$ and in Group $3(310 \pm 98 \mathrm{pg} / \mathrm{mL})$ compared to periodontally healthy subjects $(251 \pm 87 \mathrm{pg} / \mathrm{mL})(P=0.011$; KruskalWallis test). Dunn's post-hoc test showed some difference in salivary levels of hBD-2 between periodontally healthy individuals and Group 2 and between periodontally healthy individuals and Group $3(P<0.05)$; however, no difference was observed between Groups 2 and 3 , i.e., between patients with periodontal disease (Figure 1).

\section{Discussion}

Many researchers have sought to identify for biomarkers of periodontal disease to monitor its progression or facilitate its diagnosis. This study evaluated h-BD-1 and h-BD2 salivary as a possible marker of periodontal disease. Periodontal disease presents a long time consuming and expensive treatment, therefore its prevention, early detection and management are issues which, if effectively addressed, are likely to yield considerable health-care benefit. ${ }^{6}$ The presence of saliva is critical for the preservation and maintenance of the oral tissues, and it has also been used as a source of non-invasive investigation. Thus, it could contain important biomarkers related to physiological aspects of periodontal diseases, which means that qualitative changes in biomarkers composition might have diagnostic and therapeutic significance. ${ }^{18}$ Early studies focused on serum and gingival crevicular fluid samples for investigations of periodontitis related cytokines; however, in recent years, saliva has become an alternative source of biomarkers. The saliva collection has several advantages over gingival crevicular fluid: it is more easily accessible; it can be sampled in a much larger volume without the need for clinical facilities; and no complex skills are necessary for sampling. Furthermore, whereas the gingival crevicular fluid content reflects inflammatory processes at individual disease sites, it is reasonable to suggest that saliva content reflects a 'whole mouth' inflammatory status, which is likely to be much more clinically relevant. ${ }^{6}$

Studies investigating the use of saliva as a diagnostic fluid have a long history. This noninvasive approach is not limited only to the 
diagnosis of oral diseases because many systemic diseases, such as different types of cancers, cardiovascular diseases, immunologic syndromes and hereditary deficiencies, can also be studied with the aid of salivary diagnostics $(3,20,38,110)$. The continuous interaction between host cells and pathogenic bacteria are the hallmark of the development of periodontal disease. Gingival epithelial cells, mostly keratinocytes, act not only as a physical barrier against microbial factors, but also mediate the immune response by secreting antimicrobial peptides (e.g., hBD). ${ }^{19,20}$ In general, hBD1 is continuously produced by keratinocytes, ${ }^{21,22}$ but some studies have suggested that hBD-1 production can be stimulated by bacterial products and inflammatory mediators. ${ }^{23-25}$ In the present study, hBD-1 expression was observed in all samples, but patients with periodontal disease (Groups 2 and 3) had higher hBD-1 levels in their saliva when compared to their periodontally healthy counterparts (Group 1). According to Matheus et al. ${ }^{26}$ continuous contact of the oral mucosa oral with the commensal microflora can stimulate hBD-1 production even in the absence of periodontal disease; however, the presence of pathogenic microorganisms and the presence of an inflammatory process could increase hBD-1 levels. The present study did not indicate an increase in salivary levels of hBD-1 with the deterioration of periodontal disease as these levels were similar between patients with a PSR/CPITN index of 1 and 2 (Group 2) and between those with a PSR/CPITN index of 3 and 4 (Group 3); nevertheless, h-BD1 levels indicated the presence of periodontal disease since h-BD1 levels in the saliva of periodontally healthy patients (Group 1) were lower than those observed among patients with periodontal disease (Groups 2 and 3).

Regarding hBD-2 levels, this study revealed they were similar to those obtained for hBD-1. Higher concentrations were observed in Groups 2 and 3 when compared to Group 1. Albeit nonstatistically significant, hBD-1 and hBD-2 levels were slightly lower in patients with a PSR/CPITN index of 3 and 4 when compared to those with a PSR/CPITN index of 1 and 2 . We believe this small reduction in hBD1 and hBD-2 levels in the saliva occurred due to the degradation of $\mathrm{hBD}$ by proteases released at sites that are more commonly affected by periodontopathogens, as hypothesized by Yong et al. ${ }^{27}$ who verified higher hBD-2 levels in the saliva of patients with gingivitis than among those with periodontitis. Another possible explanation to this reduction could be the lower average number of teeth in Group 3

The quantification of hBD-1 and hBD-2 by ELISA has been widely used for the determination of salivary levels of these peptides. ${ }^{14,26}$ There are conflicting reports in the current literature regarding hBD-1 and hBD-2 expression in periodontal disease, as some studies suggest upregulated hBD expression ${ }^{8,14,27,28}$ whereas some suggest a down regulated expression of this peptide. ${ }^{29,30}$ In addition, previous studies found high concentrations of these peptides only after stimulation with periodonto pathogens. ${ }^{31,32}$ The difference in salivary levels of $\mathrm{hBD}$ between this and other studies could be related to the method used in the present one since the mRNA expression of hBDs measured by real-time PCR might not indicate the actual concentrations of salivary peptides..$^{33}$

Owing to the higher salivary levels of hBD-1 and hBD2 detected by ELISA in patients with periodontal disease, these antimicrobial peptides could act as potential biomarkers of periodontal disease. However, although individual salivary hBD-1 and hBD-2 levels can be statistically different between the samples obtained from periodontally healthy and periodontally compromised patients, the large variation in individual levels could prove a great hindrance to its use as biomarker of periodontal disease. ${ }^{5}$ A possible solution would be the use of diagnostic panels that combine different biomarkers. ${ }^{34}$

The lack of microbiological investigation does not allow us to determine the relationship between hBD levels and the presence of periodontopathogens and its role in the pathogenesis of periodontal disease. In conclusion, salivary levels of hBD-1 and hBD-2 were high in groups with periodontal disease detected by PSR/CPITN index when compared to the healthy group. However, the concentrations of these defensins did not vary as a result of the deterioration of periodontal condition. hBD- 1 and 2 are possible candidates as periodontal disease markers, but they cannot indicate inflammatory periodontal stages.

\section{Author contributions}

Lourenço AG and Komesu MC conceived the research; Ribeiro AERA recruited the patients and provided the samples; Lourenço AG and Ribeiro AERA performed the experiments; Lourenço AG, Komesu MC, Motta ACF and Ribeiro AERA analyzed the data; Lourenço AG, Komesu MC, Motta and Ribeiro AERA wrote the paper.

\section{Conflict of interest}

Author declared there is no conflict of interest.

\section{References}

1. Pihlstrom BL, Michalowicz BS, Johnson NW. Periodontal diseases. Lancet. 2015;366(9499):1809-1820.

2. Kim JJ, Kim CJ, Camargo PM. Salivary biomarkers in the diagnosis of periodontal diseases. J Calif Dent Assoc. 2013;41(2):119-124.

3. Papapanou PN. Systemic effects of periodontitis: lessons learned from research on atherosclerotic vascular disease and adverse pregnancy outcomes. Int Dent J. 2015;65(6):283-291.

4. Kinney JS, Ramseier CA, Giannobile WV. Oral fluid-based biomarkers of alveolar bone loss in periodontitis. Ann N Y Acad Sci. 2007;1098:230 251 .

5. Gorr SU. Antimicrobial peptides in periodontal innate defense. Front Oral. Biol 2012;15:84-98.

6. Jaedicke KM, Preshaw PM, Taylor JJ. Salivary cytokines as biomarkers of periodontal diseases. Periodontol 2000. 2016;70(1):164-183.

7. Niyonsaba F, Ushio H, Nakano N, et al. Antimicrobial peptides human beta-defensins stimulate epidermal keratinocytes migration, proliferation and production of proinflammatory cytokines and chemokines. $J$ Invest Dermatol. 2007;127(3):594-604.

8. Lourenco AG, Rodrigues Alves Ribeiro AE, Nakao C, et al. Influence of antiretroviral therapy and periodontal disease on human salivary betadefensin 2 in patients infected with HIV. Curr HIV Res. 2014;12(1):44 49.

9. Hazrati E, Galen B, Lu W, et al. Human $\alpha$ - and $\beta$-defensin block multiple steps in herpes simplex virus infection. J Immunol. 2006;177(12):86588666.

10. Weinberg A, Quinones-Mateu ME, Lederman MM. Role of human betadefensins in HIV infectious. Adv Dent Res. 2016;19(1):42-48.

11. Khurshid Z, Naseem M, Sheikh Z, et al. Oral antimicrobial peptides: Types and role in the oral cavity. Saudi Pharm J. 2016;24(5):515-524. 
12. Ali RS, Falconer A, Ikram M, et al. Expression of the peptide antibiotics human beta defensin-1 and human beta defensin-2 in normal human skin. J Invest Dermatol. 2011;117(1):106-111.

13. Harder J, Meyer-Hoffert U, Teran LM, et al. Mucoid Pseudomonas aeruginosa, TNF-alpha, and IL-1beta, but not IL-6, induce human beta-defensin-2 in respiratory epithelia. Am J Respir Cell Mol Biol. 2000;22(6):714-721.

14. Pereira AL, Franco GC, Cortelli SC, et al. Influence of periodontal status and periodontopathogens on levels of oral human $\beta$-defensin- 2 in saliva. J Periodontol. 2013;84(10):1445-1453.

15. American Dental Association and American Academy of Periodontics. Periodontal screening and recording training program kit. Chicago; 1992.

16. Ainamo J, Barmes D, Beagrie G, et al. Development of the World Health Organization (WHO) community periodontal index of treatment needs (CPITN). Int Dent J. 1982;32(3):281-291.

17. Rams TE, Loesche WJ. Relationship Between Periodontal Screening and Recording Index Scores and Need for Periodontal Access Surgery. $J$ Periodontol. 2017;88(10):1-12.

18. Gomes FI, Aragão MG, Barbosa FC, et al. Inflammatory cytokines Interleukin-1 $\beta$ and Tumour Necrosis Factor- $\alpha$-Novel biomarkers for the detection of periodontal diseases: a literature review. J Oral Maxillofac Res. 2016;7(2):e2.

19. Sorsa T, Tjaderhane L, Salo T. Matrix metalloproteinases (MMPs) in oral diseases. Oral Dis. 2004;10(6):311-318.

20. Hiroshima Y, Bando M, Kataoka M, et al. Regulation of antimicrobial peptide expression in human gingival keratinocytes by interleukin-1 $\alpha$. Arch Oral Biol. 2011;56(8):761-767.

21. Krisanaprakornkit S, Weinberg A, Perez CN. Expression of the peptide antibiotic human beta-defensin 1 in cultured gingival epithelial cells and gingival tissue. Infect Immun. 1998;66(9):4222-4228.

22. Pernet I, Reymermier C, Guezennec A, et al. Calcium triggers betadefensin (hBD2 and HBD-3) and chemokine macrophage inflammatory protein-3 $\alpha$ (MIP-3alpha/CCL20) expression in monolayers of activated human keratinocytes. Exp Dermatol. 2003;12(6):755-760.

23. Sahasrabudhe KS, Kimball JR, Morton TH. Expression of the antimicrobial peptide, human beta-defensin 1, in duct cells of minor salivary glands and detection in saliva. J Dent Res. 2000;79(9):16691674.
24. Dale BA. Periodontal epithelium: a newly recognized role in health and disease. Periodontology. 2002;30:70-78.

25. Prado-Montes de Oca E. Human beta-defensin 1: a restless warrior against allergies, infections and cancer. Int $J$ Biochem Cell Biol. 2010;42(6):800-804

26. Mathews M, Jia HP, Guthmiller JM, et al. Production of beta-defensin antimicrobial peptides by the oral mucosa and salivary glands. Infect Immun. 1999;67(6):2740-2745.

27. Yong X, Chen Y, Tao R, et al. Periodontopathogens and human $\beta$-defensin-2 expression in gingival crevicular fluid from patients with periodontal disease in Guangxi, China. $J$ Periodontal Res. 2015;50(3):403-410.

28. Pereira AL, Holzhausen M, Franco GCN, et al. Human beta-defensin 2 and protease activated receptor- 2 expression in patients with chronic periodontitis. Arch Oral Biol. 2012;57(12): 1609-1614.

29. Vardar-Sengul S, Demirci T, Sen BH, et al. (2007) Human beta defensin-1 and -2 expression in the gingiva of patients with specific periodontal diseases. J Periodontal Res. 2007;42(5):429-437.

30. Dommish H, Acil Y, Dunsche A, et al. Differential gene expression of human beta-defensins (hBD-1-2-3) in inflammatory gingival diseases. Oral Microbiol Immunol. 2005;20(3):86-190.

31. Ouhara K, Komatsuzawa H, Shiba H, et al. Actinobacillus actinomycetemcomitans outer membrane protein 100 triggers innate immunity and production of beta-defensin and the 18-kilodalton cationic antimicrobial protein through the fibronectin-integrin pathway in human gingival epithelial cells. Infect Immun. 2006;74(9):5211-5220.

32. Noguchi T, Shiba H, Komatsuzawa H, et al. Syntheses of prostaglandin E2 and E-cadherin and gene expression of beta-defensin-2 by human gingival epithelial cells in response to Actinobacillus actinomycetemcomitans. Inflammation. 2003;27(6):341-349.

33. Hosokawa I, Hosokawa $\mathrm{Y}$, Komatsuzawa $\mathrm{H}$, et al. Innate immune peptide LL-37 displays distinct expression pattern from beta-defensins in inflamed gingival tissue. Clin Exp Immunol. 2006;146(2):218-225.

34. Miller CS, Foley JD, Bailey AL, et al. Current developments in salivary diagnostics. Biomark Med. 2010;4(1):171-189. 